Original Paper http://ajol.info/index.php/ijbcs $\quad$ http://indexmedicus.afro.who.int

\title{
Dynamique de la mangrove du marigot de Bignona autour du barrage d'Affiniam (Casamance, Sénégal)
}

\author{
Mariétou TENDENG ${ }^{1 *}$, Ngor NDOUR ${ }^{1}$, Bienvenu SAMBOU $^{2}$, Malaïny DIATTA $^{3}$ \\ et Alain AOUTA ${ }^{4}$ \\ ${ }^{1}$ Département d'Agroforesterie, Unité de Formation et de Recherche en Sciences et Technologie, Université \\ Assane Seck de Ziguinchor, BP 523 Ziguinchor, Sénégal. \\ ${ }^{2}$ Institut des Sciences de l'Environnement, Faculté des Sciences et Techniques, Université Cheikh Anta Diop \\ de Dakar, BP 5005 UCAD Dakar, Sénégal. \\ ${ }^{3}$ ISRA/CNRF, Bp: 2312 Dakar ; Sénégal.Tel : 7756631 40, Email :djinkadiatta@yahoo.fr \\ ${ }^{4}$ Association OCEANIUM, projet D'évaluation du Carbone des Formations Forestières, Sénégal. \\ Tel:+221779635926 \\ *Auteur correspondant : E-mail: marietou_tendeng@yahoo.fr
}

\section{RESUME}

La sècheresse des années 1970 a contribué à la dégradation de la mangrove en Casamance. Les activités socio-économiques (pêche, riziculture...) sont devenues de moins en moins rentables. Pour faire face aux conséquences des aléas climatiques, le barrage d'Affiniam fut construit sur le marigot de Bignona. Cependant, à la suite de sa construction, la dégradation de la mangrove a semblé s'accélérer en amont comme en aval du barrage. Dans ce contexte, la présente étude tente de comprendre cette dégradation ainsi que la dynamique de l'écosystème autour du barrage. A cet effet, des cartes d'occupation des sols ont été confectionnées avant et après la construction du barrage. Des relevés de végétation ont été faits le long d'un transect de $12 \mathrm{~km}$ passant par le barrage. Les résultats montrent que la mangrove a régressé de 55,58\% entre 1986 et 2010 autour de l'ouvrage. Sa densité actuelle est de 3869 individus/ha en aval et de 245 individus/ha en amont. La mangrove de la partie aval est moins touchée par la dégradation. Cependant, la salinité des eaux peut y atteindre $120 \mathrm{~g} / \mathrm{l}$ en fin de saison sèche, alors que le pH varie entre 6,5 et 7,5. Malgré ce taux de salinité élevé, le pH oscille autour de la neutralité $(\mathrm{pH}=7)$.

() 2016 International Formulae Group. All rights reserved.

Mots clés: Dégradation, régression, amont, aval, Casamance.

\section{ABSTRACT}

Dynamics of the mangrove ecosystem around the Affiniam dam (Casamance, Senegal) on the Bignona backwater. The drought in the 1970s has contributed not only to the mangrove system degradation but also to the decrease of socio-economic profits for rural communities (fishing, rice production...). To accommodate to the adverse weather conditions, the Affiniam dam was built on the backwater of Bignona. However, its construction significantly reduced and degraded the mangrove system affecting the upstream as well as the downstream parts of the dam. To understand the causes of the environmental deterioration and the dynamics of the ecosystem around the dam, maps of the lands used before and after construction of the dam were made and vegetation inventory were conducted along a $12 \mathrm{~km}$ transect across it. The results of this study showed that the mangroves decreased by $55.58 \%$ between 1986 and 2010 in the area surrounding the dam with a current 
density of 3869 individuals/ha downstream and 245 individuals/ha upstream. The downstream portion of the mangroves where the tides are still effective is less affected by the process of environmental deterioration. However, water salinity can reach $120 \mathrm{~g} / \mathrm{l}$ at the end of the dry season while water $\mathrm{pH}$ can vary between 6,5 and 7,5. Despite this high rate of salinity, the $\mathrm{pH}$ oscillates around 7 (neutral $\mathrm{pH}$ ).

(C) 2016 International Formulae Group. All rights reserved.

Keywords: Degradation, regression, upstream, downstream, Casamance.

\section{INTRODUCTION}

La sécheresse observée pendant les années 1970 en zone sahélienne a affecté des régions humides comme la basse Casamance au sud du Sénégal (Albergel et al., 1991 ; Niasse et al., 2004). Le déficit pluviométrique a participé à la dégradation des écosystèmes côtiers au Sénégal (Ndao, 2012 ; Dieye et al., 2013) et a favorisé un accroissement de la salinité des eaux dans le fleuve et la baisse du niveau des nappes phréatiques (Montoroi, 1996 ; Ndao, 2012 ; Ndiaye, 2003, Bassène, 2010 ; Dasylva, 2013). En Casamance, les activités telles que la pêche, la collecte de bois, l'ostréiculture... liées à l'exploitation de la mangrove et celles liées à la riziculture souvent pratiquée en arrière-plan ont vu leur rentabilité nettement en baisse. Cette situation a contribué à l'exode rural des populations vers les centres urbains (Montoroi et Zante, 1989 ; Fall, 2004). Pour trouver une solution aux aléas climatiques, des ouvrages hydroagricoles ont été édifiés au début des années 1980 au Sénégal parmi lesquels la construction des barrages anti-sel de Guidel et d'Affiniam en Casamance. L'hypothèse était fondée sur la récupération et l'aménagement de vastes superficies de bas-fonds dans une région jugée capable de dégager un surplus de production rizicole pour contribuer à la sécurité alimentaire du pays (Keita, 2004 ; Diouf, 2013 ; Ndiaye, 2004). Ces ouvrages, associés à des évacuateurs et à des réseaux de drainage par fossés, devaient permettre le dessalement des zones protégées après quelques saisons de pluies et la retenue d'eau douce pour l'irrigation (Bosc 2005, cité par Diouf, 2013). Le barrage d'Affiniam, fruit de la coopération entre le Sénégal et la République Populaire de Chine, est érigé sur le marigot de Bignona qui est un affluent de la rive droite du fleuve Casamance. Il avait pour objectifs principaux de stopper la progression du sel, de contribuer au dessalement des rizières et de permettre la mise en valeur de plus de 12000 ha de terres agricoles (Barry et al., 1988;). Cependant, si la construction de ce barrage constituait à priori une solution pour le développement de la riziculture, elle semble être aujourd'hui la cause de l'accélération de la dégradation de la mangrove dans la zone d'étude (Manga et Tendeng, 2010; Fall, 2004). La présente étude a été menée pour mieux comprendre la dynamique de la mangrove autour du barrage afin de contribuer à une meilleure adaptation des populations aux effets positifs ou négatifs de l'ouvrage.

\section{MATERIEL ET METHODES}

Le barrage d'Affiniam se trouve dans la communauté rurale de Mangagoulack qui est une composante de l'arrondissement de Tendouck, lui-même localisable dans le département de Bignona. Il est construit dans un site relativement enclavé, situé à 25 kilomètres de Bignona, qui est un département de la région de Ziguinchor (Keita, 2004). Ce département est situé à une trentaine de kilomètres au nord de Ziguinchor, entre les coordonnées géographiques $12^{\circ} 49^{\prime} \quad \mathrm{N}$ et $16^{\circ} 14^{\prime}$ W (A.U.O.F, 2000, PADERCA, 2008). La zone d'étude s'étend sur un rayon de six (6) $\mathrm{km}$ à partir du barrage d'Affiniam. Ce rayon délimite la zone qui englobe cinq villages dont trois (Baghagha, Diagobel et Affiniam) en aval du barrage et deux 
(Diandialate et Kandiou) en amont (Figure 1). Le climat de la zone est de type soudanien avec une température moyenne de $27^{\circ}$ (ANSD 2013). Il est marqué par l'alternance d'une saison sèche et d'une saison des pluies. La première dure 7 mois (Novembre à Mai) et la seconde 5 (Juin à Octobre). Plus de la moitié des précipitations se concentre entre Juillet et Septembre mais très irrégulièrement réparties. Les populations, dont le niveau de vie est bas, mènent comme principales activités socioéconomiques l'agriculture, la pêche, et l'élevage. Néanmoins, d'autres activités telles que le commerce, l'exploitation forestière et le maraîchage étaient également menées. (Manga et Tendeng, 2010 ; Fall, 2004).

\section{Matériel}

Divers matériels ont permis de faire l'inventaire de la mangrove. Les spatio-cartes et un GPS Map 60 ont permis de déterminer l'axe du transect et l'accès rapide aux placettes d'inventaire. Ces dernières ont été délimitées à l'aide de quatre jalons et d'une corde. Les fiches de collectes de données ont servi à l'inventaire de la flore et de la végétation de la mangrove. Un ruban métrique de 50 mètres a été utilisé pour mesurer les dimensions des placettes d'inventaire. Les consignes de sécurité adoptées par les membres de l'équipe d'inventaire reposent sur le port de gilets, l'utilisation de la boussole du GPS et du téléphone portable. Les autorités de la police locale étaient aussi informées de la présence de l'équipe dans la mangrove...) Des logiciels de traitement d'images (ArcGis 9.2, ERDAS Imagine 8.1, Global Mapper 8. 2, Mapsource) ont permis la réalisation des spatio-cartes et des cartes de la dynamique de la mangrove autour du barrage.

\section{Collecte de données images et choix du pas de temps}

Les données images disponibles pour la réalisation du travail étaient des capteurs TM et ETM+ de la génération des satellites
Landsat (1 à 7) dont la résolution en mode multi spectrale est de $30 \mathrm{~m}$. Ce choix est lié à la fois à un besoin d'analyse diachronique (avant barrage) et synchronique (après barrage). L'analyse diachronique se réfère à l'image Landsat de 1986 qui coïncide avec le démarrage de la construction du barrage. Elle a été choisie pour établir la situation de référence de l'état de la mangrove avant la mise en place du barrage. Avec un "pas" de 24 ans entre elles, les deux images ont permis de faire un état des lieux de la mangrove et de montrer les changements intervenus dans l'écosystème par la production de cartes d'occupation des sols avant et après barrage.

\section{Préparation des missions de terrain}

Un transect d'une longueur de 12 kilomètres allant de l'aval vers l'amont du barrage a été élaboré pour faire l'état des lieux des formations végétales dans la zone d'étude. Vingt-quatre (24) placettes de taille $10 \mathrm{~m} \mathrm{x} 10$ $\mathrm{m}$ chacune ont été installées de part et d'autre du transect. L'équidistance entre les placettes d'inventaires est de $500 \mathrm{~m}$, ce qui a permis de mieux les répartir sur le transect (Figure 2). Les placettes ont été positionnées sur la spatio-carte géoréferencée dont le fond est une image google Earth et les coordonnées de leur centre ont été extraits automatiquement à l'aide du logiciel ArcGis 9.2, puis converties au format GPX à l'aide du logiciel Global mapper 8.2. Ces coordonnées ont permis de retrouver les placettes d'inventaire sur le terrain à l'aide d'un GPS.

\section{Relevés de végétation}

Lors des relevés de végétation, les placettes d'inventaire ont été délimitées à l'aide d'un ruban métrique, d'une corde et de quatre jalons. Dans chaque placette, les espèces végétales présentes sont identifiées à l'aide de la clé de Berhaut (1967), et la régénération naturelle est comptée. La hauteur de chaque individu a été mesurée à l'aide d'un jalon gradué ou d'un décamètre. Le taux de 
recouvrement a été estimé en projetant la couronne des individus au sol dans chaque placette. Les espèces animales présentes ou rencontrées ont été notées. Des mesures de salinité et de $\mathrm{pH}$, des eaux du marigot, ont été effectuées de l'amont vers l'aval du barrage.

\section{Prétraitement des images satellitaires acquises}

Les images satellitaires ont subi un certain nombre de traitements ayant permis de cartographier les changements intervenus autour du barrage entre 1986 et 2010. Elles ont été calées à l'aide d'une image de référence de la zone déjà géoréferencées. Quatorze (14) amers (points homologues entre l'image de référence et celles acquises (TM et ETM+) ont été choisies. La méthode de rééchantillonnage est le plus proche voisin et la correction géométrique a été effectuée selon une transformation polynomiale du premier ordre. Le contrôle de la validité s'est fait par l'estimation de l'erreur standard, RMSE (Root Mean Square Error) ou Erreur Moyenne Quadratique. La RMSE n'étant qu'un indicateur, la validation des corrections géométriques a été complétée par un contrôle visuel effectué par la superposition des images. Le seuil de précision spatiale retenu étant une erreur de moins d'un pixel. Elles ont été ensuite rehaussées par étalement de la dynamique et des compositions colorées fausse couleur (4-3-2) ont été créés pour une meilleure interprétation des thèmes de l'image. Le choix des canaux a été basé sur le proche infrarouge (canal 4) parce que la végétation réfléchie mieux dans ce canal, et les deux canaux du visible le rouge (canal 3) et le vert (canal 2).

\section{Traitement des images : Classification supervisée}

La technique utilisée pour la mise en évidence des changements intervenus autour du barrage repose sur la méthode de la classification supervisée avec comme méthode d'interpolation le maximum de vraisemblance. La classification supervisée consiste à regrouper les unités de paysages ayant la même réponse spectrale en une seule classe dite «classe thématique ». Pour avoir une classe thématique on collecte partout dans l'image des zones d'intérêt appelées parcelles d'entraînement. Ces parcelles sont choisies de manière à ce qu'elles présentent la même radiométrie et ensuite sont regroupées en une seule classe dite thématique automatiquement par le logiciel sous la commande de l'opérateur. Elle nécessite une interprétation visuelle de l'image basée sur la connaissance des signatures spectrales de l'ensemble des éléments qui la composent. Ce traitement a permis d'obtenir une image dite classifiée, constituée de plusieurs classes thématiques de 1986 et 2010 respectivement des capteurs TM et ETM+ du satellite Landsat.

\section{Traitement des données Floristiques}

Par ailleurs, les données floristiques ont été traitées à l'aide du logiciel Excel. Le taux de recouvrement moyen de la végétation ligneuse a été calculé en aval comme en amont du barrage. La densité de la végétation qui correspond au nombre total d'individus par unité de surface a été calculée suivant la relation : $D=\frac{i \mathbf{T}}{\xi}$ où $N t$ est le nombre total d'individus et $S$ la superficie inventoriée. La fréquence relative des espèces a été calculée suivant la relation : $F_{r}=\frac{F_{e}}{F_{t}} \times 100$ avec Fe étant la fréquence d'une espèce et Ft le total des fréquences de toutes les espèces inventoriées. La densité relative des espèces ligneuses a été calculée à l'aide de la formule suivante: $D r=\frac{n e}{N t} * 100$ où ne est le nombre d'individus d'une espèce et $\mathrm{Nt}$ le nombre total d'individus de toutes les espèces inventoriées. Dans le cadre de l'analyse de la structure de la végétation, la hauteur des espèces ligneuses a été mesurée. Le "pas" 
entre les classes de hauteur est de $1 \mathrm{~m}$ et la classe] 0 1] regroupe les palétuviers appartenant à la régénération naturelle. Ces différents paramètres et méthodes de traitement ont permis d'analyser la dynamique de la mangrove et de faire des comparaisons des différentes situations entre la partie amont et la partie aval avant et après la construction du barrage.

\section{RESULTATS}

\section{Évolution de la superficie de la mangrove}

Avant la construction du barrage, la carte d'occupation des sols de 1986 montre que la mangrove était présente de part et d'autres de sa position actuelle. La superficie totale des palétuviers était de 4203,99 ha dont $22,75 \%$ en amont et $77,24 \%$ en aval de la position actuelle de l'ouvrage (Figure 3). Après la mise en place de celui-ci, la carte d'occupation des sols de 2010 montre que la superficie de la mangrove est réduite à 1867,68 ha soit une baisse de 55,58\% par rapport à la situation de 1986 . En 2010, il ne restait plus que 201,78 ha de mangrove en amont et 1665,9 ha en aval du barrage. Entre les deux dates, la mangrove a régressé de $78,9 \%$ en amont et de $48,7 \%$ en aval. En conséquence, la formation des tannes a été plus importante en amont du barrage. La superficie de la mangrove a subit une régression après la construction du barrage, surtout en amont où la mangrove a quasiment disparu. La mise en place du barrage d'Affiniam semble être la cause principale de l'extension des tannes dans la partie amont.

Évolution de la flore et la végétation de la mangrove

Dans le cadre de l'analyse de la flore et de la végétation réalisée en 2013 , les espèces de palétuviers présentes dans la zone d'étude sont composées de Rhizophora mangle L., Rhizophora racemosa G.F. Mey., Rhizophora harisonnii Leech., Avicennia germinans P. Beauv., Conocarpus erectus L. et Laguncularia racemosa Gaertn. En amont du barrage, seules Avicennia germinans et Rhizophora mangle sont rencontrées. Les espèces herbacées sont principalement Sesuvium portulacastrum L. et Philoxerus vermicularis (L.) P. Les eaux douces stockées en amont ont favorisé le développement de nouvelles espèces végétales telles que Nymphö̈des indica (L.) O. Kze., Nymphaea lotus L., Fuirena ciliaris Roxb., Imperata cylindrica Beauv. et Vernonia colorata (Willd.) Drake. La densité de la végétation est de 3869 individus/ha en aval et de 245 individus/ha en amont. Il apparaît aussi que la densité est 16 fois plus importante en aval qu'en amont du barrage. Absents de la partie amont, les individus de la régénération naturelle présentent une densité de 2154 individus/ha en aval du barrage. Les densités relatives de Rhizophora mangle, Avicennia germinans, Vernonia colorata et Rhizophora harissonii sont respectivement de 76,6\%, de $18,3 \%, 4,9 \%$ et de 0,2\% (Figure 6). Les fréquences relatives de Rhizophora mangle et Avicennia germinans sont respectivement de $60 \%$ et $30 \%$, alors que Rhizophora harissonii et Vernonia colorata présentent chacune 5\% (Figure 7).

\section{Physionomie et structure de la végétation autour du barrage}

Le taux de recouvrement de la végétation est faible dans la zone d'étude. Il est de $10,8 \%$ en aval et $0,8 \%$ en amont. Bien que faible, en aval du barrage, il est 13,5 fois plus important que dans la partie amont (Figure 5). La hauteur moyenne des espèces ligneuses est de 3,09 $\mathrm{m}$ avec des pics de $36 \mathrm{~m}$. Toutefois, l'analyse de la structure verticale de la végétation montre l'importance des individus et leur catégorisation dans les différentes classes. Ainsi, la classe de hauteur [0-1] représente la régénération naturelle, celles de hauteur ]1-2]; ]2-3] et 13-4] correspondent à la mangrove de hauteur moyenne. Ces trois classes sont les plus représentatives de la zone d'étude. Les classes de hauteur ] 4-5] et 15-6] correspondent aux individus adultes rencontrés près des bolongs. Les classes de hauteur ] 30-31] et] 35-36] renferment les individus rares. Le vide noté entre la classe 15-6] et la classe ]28-29] 
montre que la structure verticale de la végétation est discontinue. L'espèce Vernonia colorata, présente dans la zone est rencontrée dans la partie amont du barrage. Il s'agit d'une espèce continentale relativement halotolérante qui pousse sur les îlots sableux (Figure 4).

\section{Salinité et acidité de l'eau dans la zone}

La salinité des eaux du marigot est plus élevée en aval du barrage qu'en amont. Elle est comprise entre 38 et $120 \mathrm{~g} / \mathrm{l}$ en aval et entre 7 et $14 \mathrm{~g} / \mathrm{l}$ en amont (Figure 9). Quant à la variation du $\mathrm{pH}$ des eaux du marigot, elle est faible entre la partie amont et la partie aval. En moyenne, le pH est de 7,25 en aval et de 6,7 en amont du barrage pendant le mois de mai de l'année 2013. Cependant, il est à préciser que le $\mathrm{pH}$ a évolué entre 7 et 7,5 en aval alors qu'il a fluctué entre 6,5 et 6,99 en amont du barrage. Ces statistiques laissent présager des conditions environnementales plus favorables à la vie aquatique dans la partie aval que dans la partie amont de l'ouvrage.

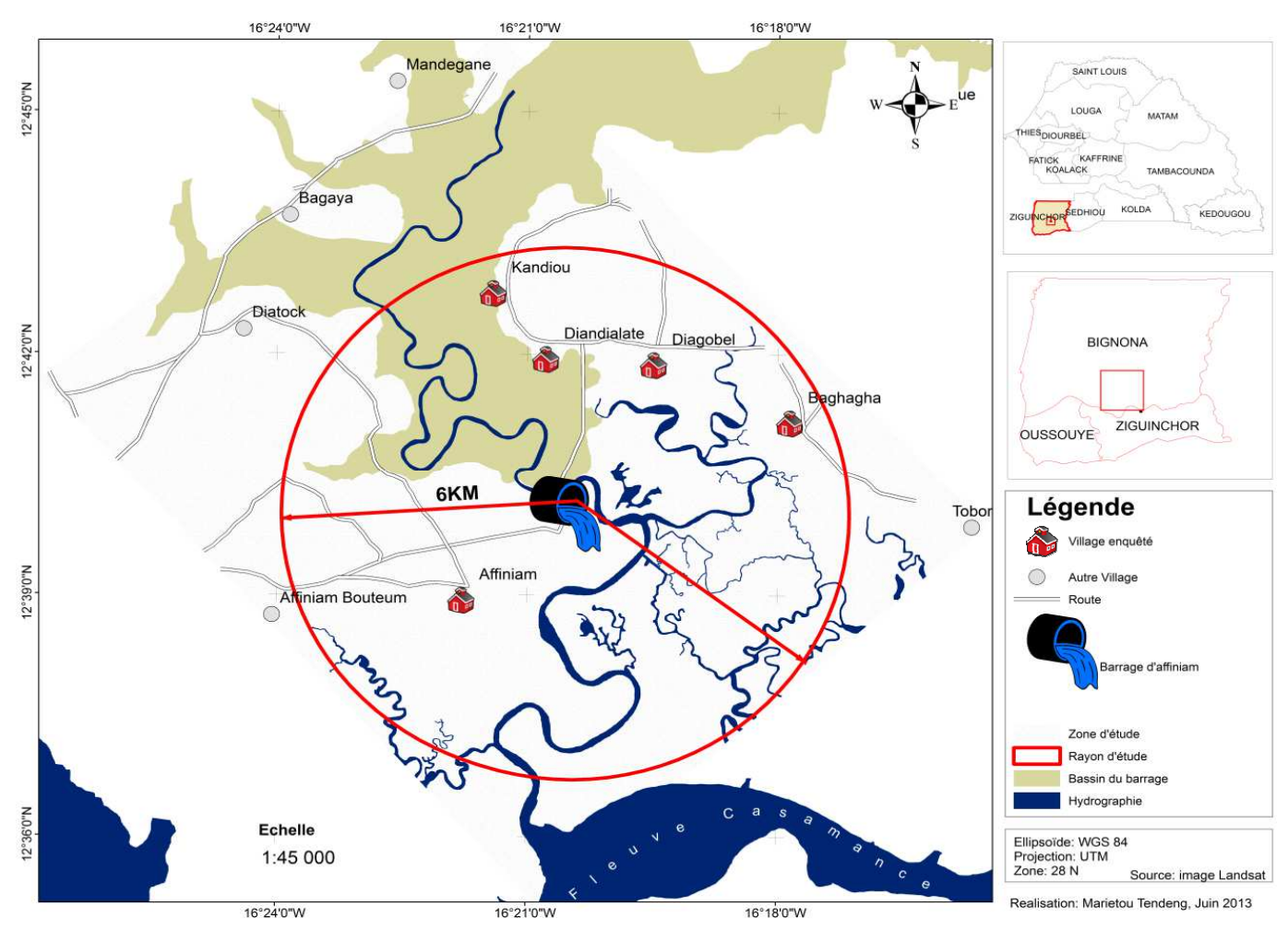

Figure 1: Carte de localisation de la zone d'étude. 


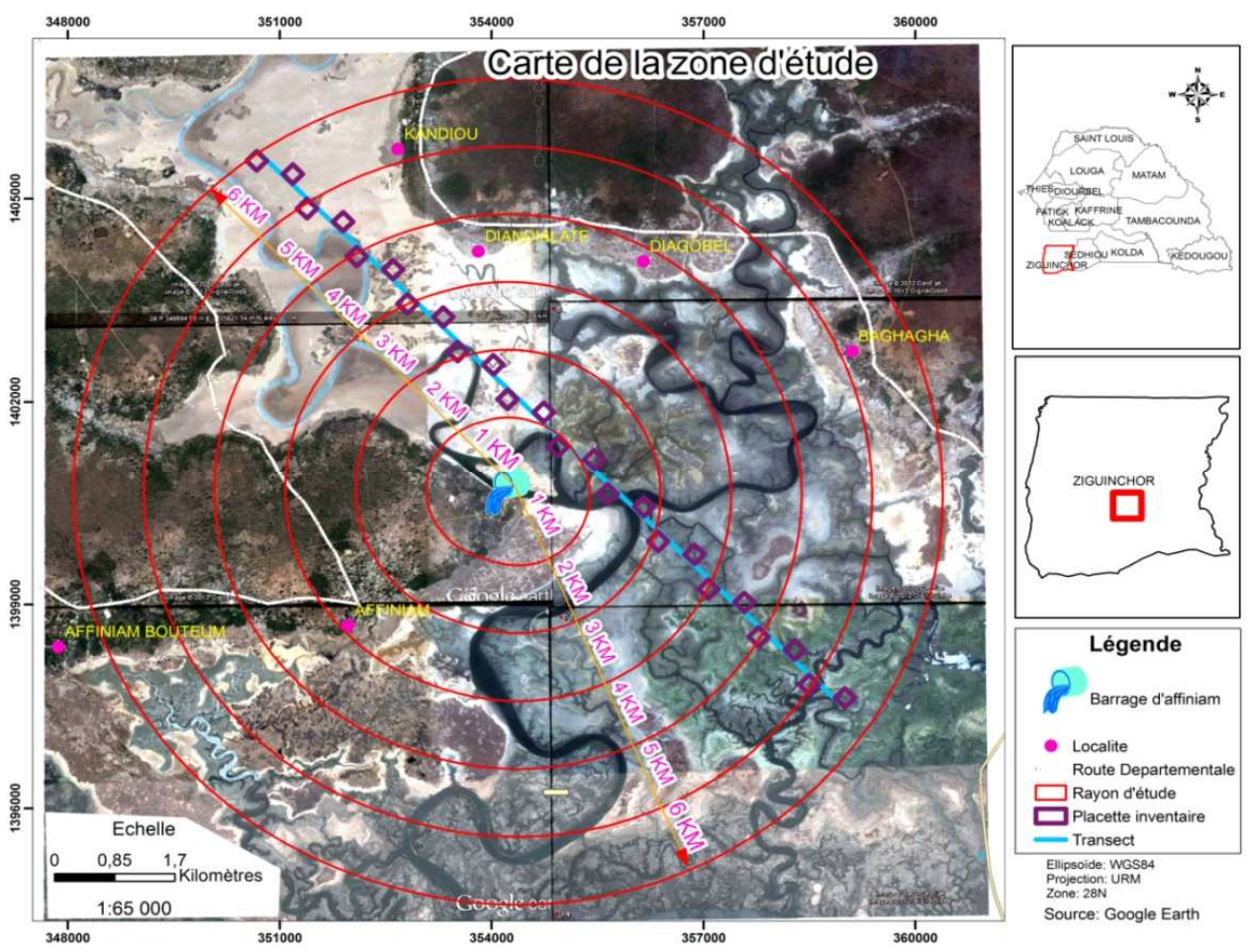

Figure 2: Placettes d'inventaire de la zone d'étude.
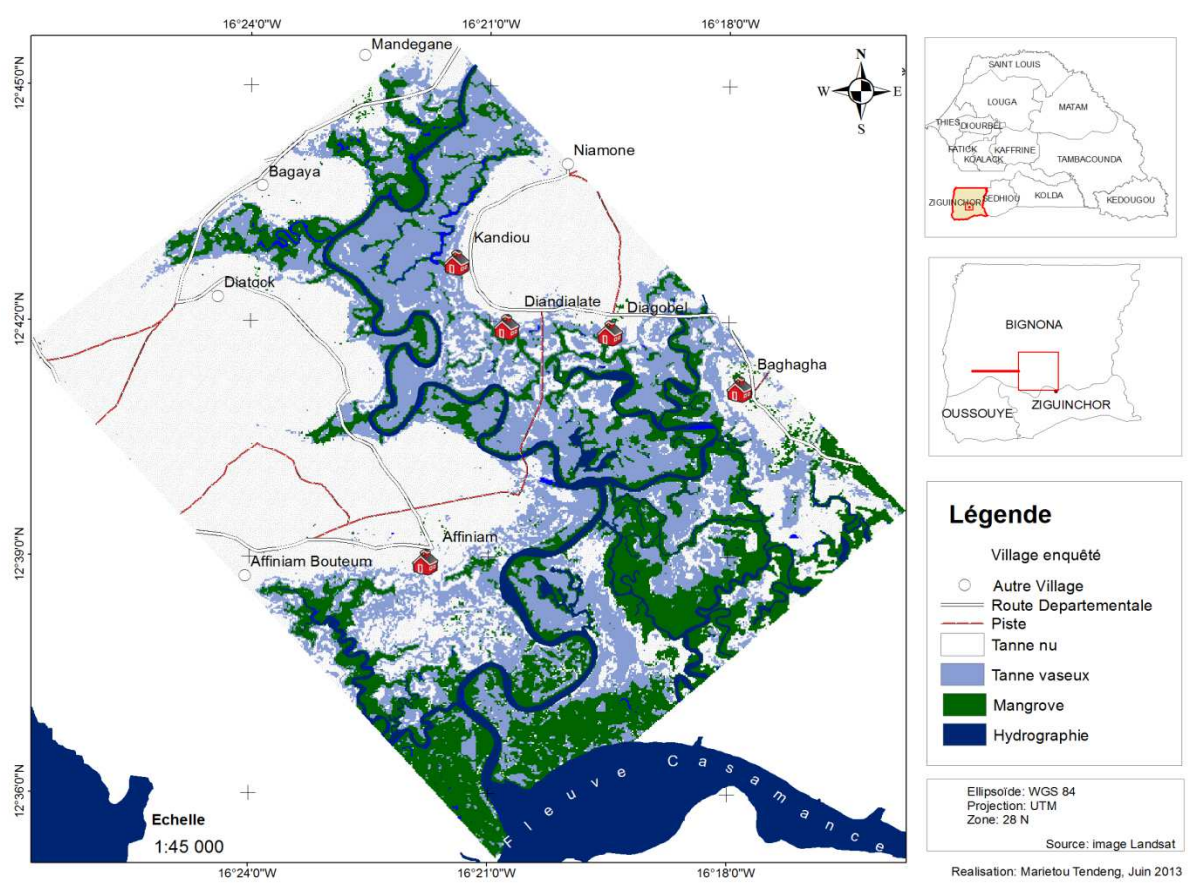

Figure 3: Carte d'occupation des sols de la zone d'étude avant l'achèvement du barrage en 1986. 

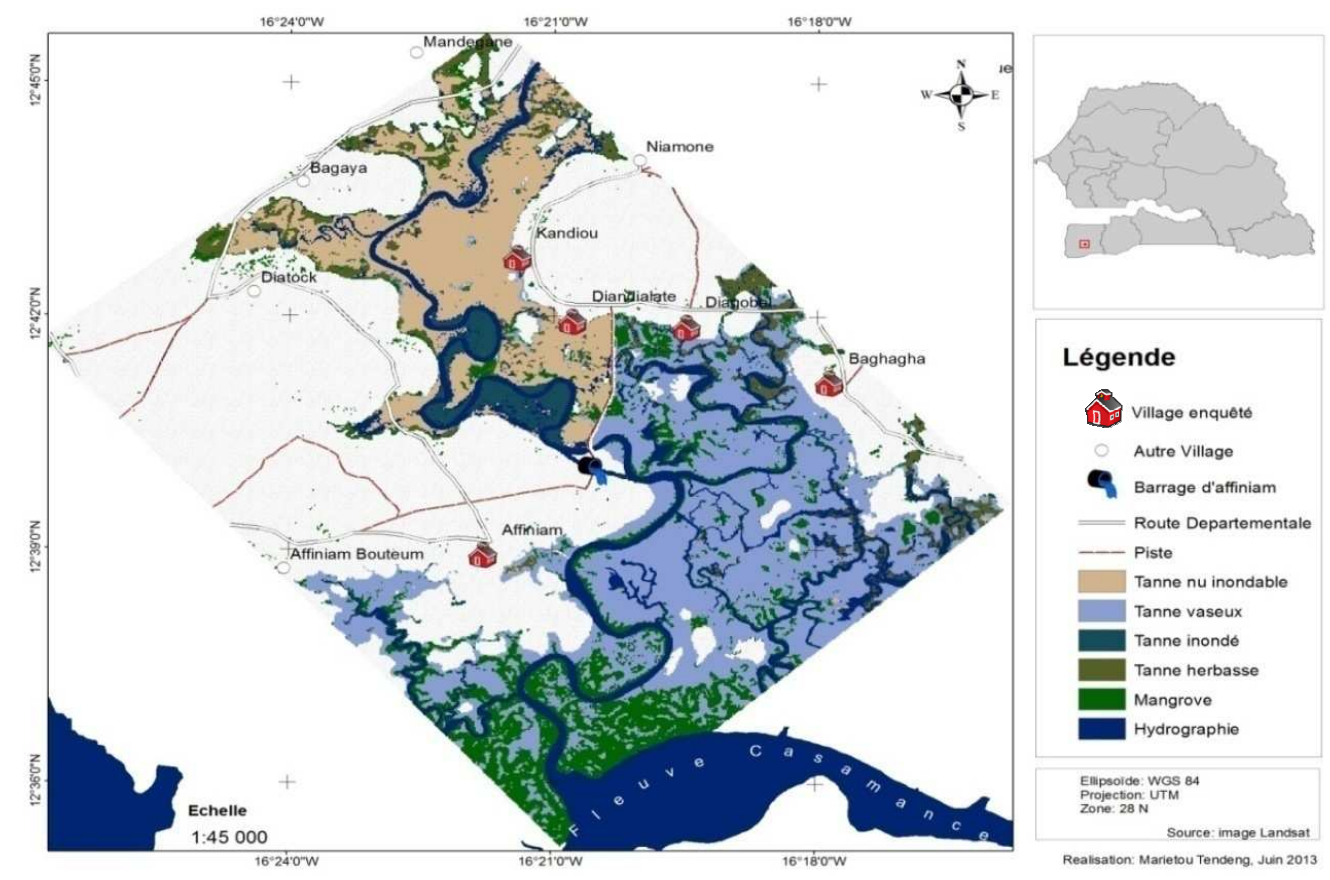

Figure 4: Carte d'occupation des sols de la zone d'étude après la construction du barrage en 2010.

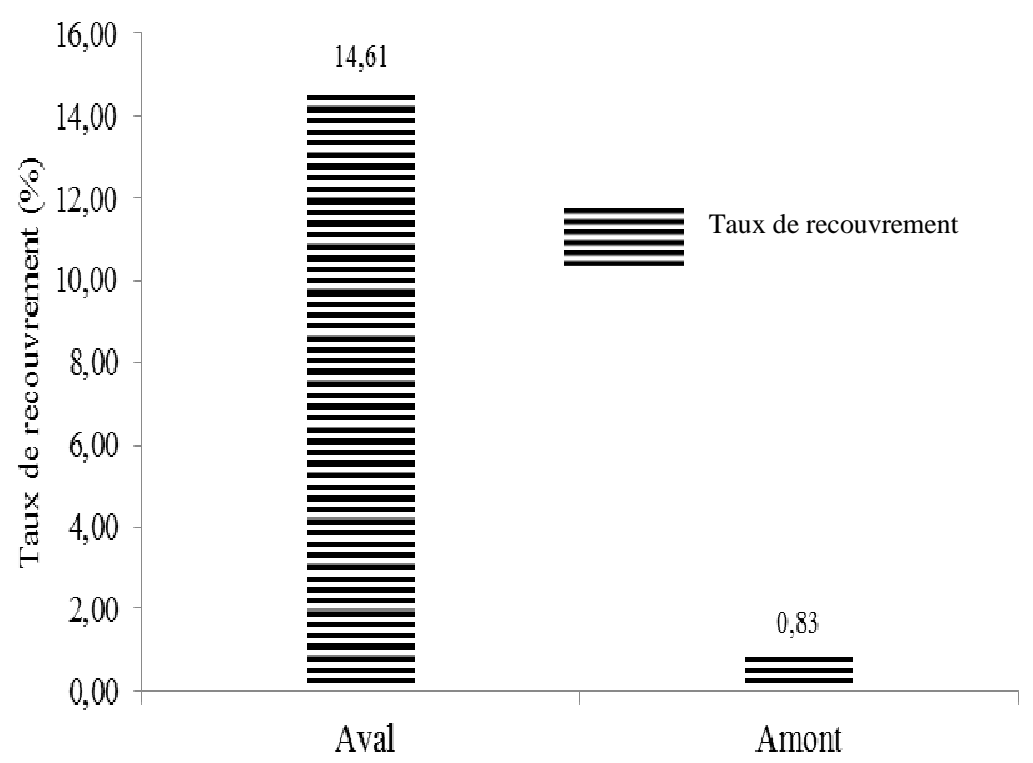

Figure 5: Taux de recouvrement des espèces ligneuses. 


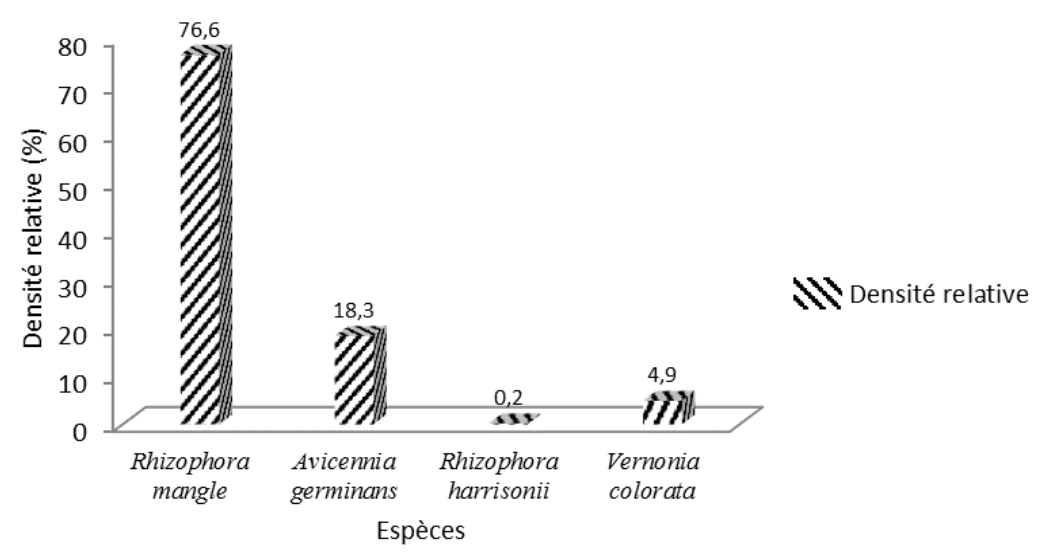

Figure 6: Densité relative des espèces ligneuses.

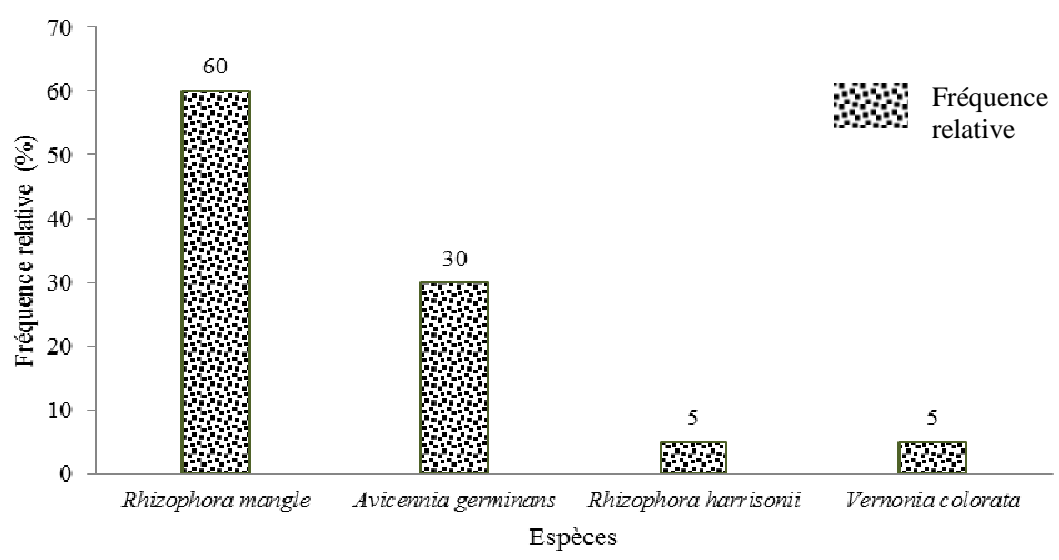

Figure 7: Fréquence relative des espèces ligneuses autour du barrage.

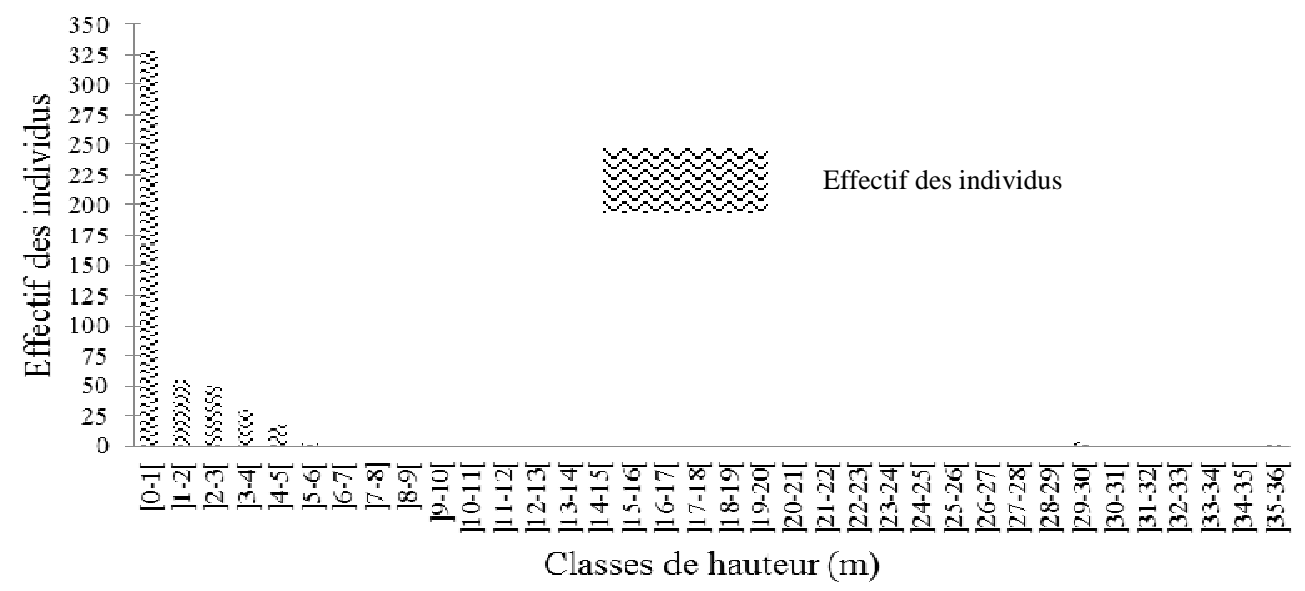

Figure 8: Répartition du nombre d'individus par classe de hauteur. 


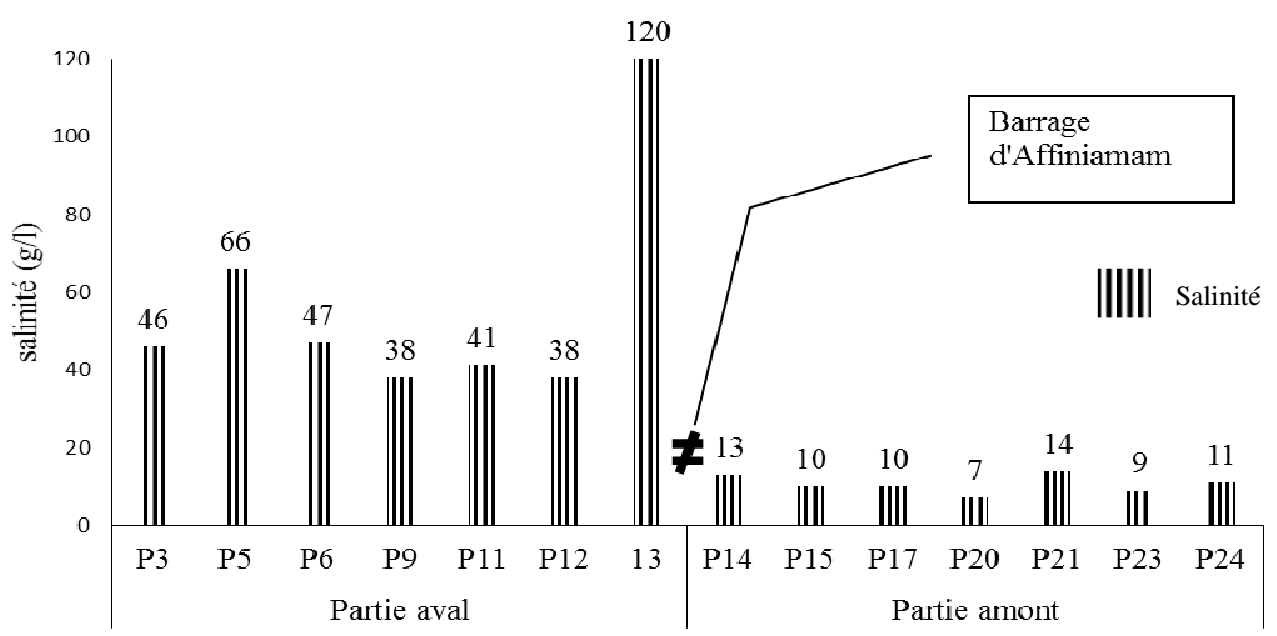

Figure 9: Salinité des eaux du marigot autour du barrage d'Affiniam.

\section{DISCUSSION}

Effet du barrage sur la mangrove du marigot

Le présent travail a permis de montrer qu'avant la construction du barrage, la mangrove était présente le long du marigot de Bignona, notamment dans les villages d'Affiniam, Baghagha, Diagobel, Kandiou et Diandialate. D'ailleurs, plusieurs auteurs avaient signalé la présence de la mangrove dans ces zones les premières années après la construction du barrage (Fall, 2004 ; Fermont, 2002). Cette situation pourrait s'expliquer par le fait que les effets négatifs du barrage ce sont manifestés progressivement sur la mangrove. Au fur et à mesure, la mangrove s'est dégradée des deux côtés du barrage La perte importante de superficie de la mangrove dans la partie amont du barrage ainsi que l'extension des tannes peuvent s'expliquer en partie par la modification du régime hydrologique du cours d'eau et la submersion plus longue des palétuviers (Figure 5). En effet, pendant la saison des pluies, les terres de la partie amont sont submergées par les eaux douces. Cette nouvelle situation hydrologique a contribué à la mort des palétuviers à cause de leur immersion continue pendant plus de trois mois. Du fait de l'assèchement de la partie amont pendant la saison sèche, le facteur acidité du sol a dû aussi contribuer à la dégradation de la mangrove en amont. Cette hypothèse est en phase avec les résultats de divers auteurs notamment Manga et Tendeng, (2010), Fall (2004). Ces auteurs soutiennent que la mise en place du barrage a entraîné des modifications profondes de l'environnement autour du barrage. Selon eux et selon Ndiaye (2003), la retenue d'eau douce crée un milieu écologique très différent du milieu aquatique initial. D'après le CSE (2010), la perturbation des régimes hydriques, de submersion constitue une cause de dégradation des formations végétales des écosystèmes côtiers. En effet, après l'installation du barrage, les sols de la partie amont ne sont plus inondés au rythme des marées mais selon l'ouverture et la fermeture des vannes du barrage. Ces sols restent inondés pendant la saison des pluies et exondés à certains endroits en pleine saison sèche, ce qui a contribué à la mort des palétuviers et favorisé la formation de tannes dans cette partie. A cet effet, certains auteurs ont montré que l'exondation prolongée des sols initiaux de mangrove provoque des modifications physicochimiques et morphopédologiques marquées par une forte 
salinisation et une acidification des sols (Dieye et al., 2013). Ces modifications entraînent aussi une disparition progressive de la mangrove et la formation de tannes (Dieye et al., 2015). Ces propos sont soutenus par les auteurs tels que Assani et petit (2004), UICN (2009), Rollet (2007). Selon ces auteurs, les barrages ont des impacts sur la faune et la flore des cours d'eau. Ces impacts sont principalement liés au blocage des migrations piscicoles (Schilt, 2007), aux modifications des nutriments, de la température de l'eau, de la charge de fond (Daube et al., 2003).

\section{Effet du barrage sur la salinité et l'acidité de l'eau du marigot}

Les effets des barrages sur l'équilibre dynamique et le fonctionnement des cours d'eau ont été largement étudiés et illustrés selon Brandt (2000a, 2000b) Les mesures de salinité effectuées montrent une variation du taux de salinité de l'aval vers l'amont du barrage. La salinité est plus élevée en aval du barrage où elle est comprise entre 38 et 120 $\mathrm{g} / \mathrm{l}$ et entre 7 et $14 \mathrm{~g} / \mathrm{l}$ en amont au mois de mai 2013. Cette différence peut s'expliquer par le fait que le barrage a limité les échanges d'eaux entre la partie amont et la partie aval. En effet, après l'achèvement des travaux du barrage en 1988, l'eau salée ne remonte plus en amont de l'ouvrage car les vannes sont maintenues fermées en saison sèche. Pendant cette période, la concentration en sel des eaux de la partie amont augmente avec l'assèchement du cours. Par ailleurs, le sel accumulé dans la partie amont pendant la saison sèche est lessivé et transporté vers l'aval au cours des lâchées d'eau pendant la saison des pluies. Au mois d'octobre, la salinité variait entre 20 et $21 \mathrm{~g} / \mathrm{l}$ dans la partie aval et entre 2 et $5 \mathrm{~g} / \mathrm{l}$ en amont (Manga et Tendeng. 2010). Les écarts notés entre ces deux dates pourraient s'expliquer d'une part par le fait que les mesures ont été réalisées à des périodes différentes de l'année. Au mois d'octobre, les eaux douces de la saison des pluies contribuent à la baisse de la salinité des eaux du marigot. Au mois de mai, la salinité des eaux de la partie amont augmente sous l'effet de l'évaporation et/ou du dessèchement observé à certains endroits. En aval du barrage, l'évaporation devient plus importante et exacerbe la salinité des eaux. Ces résultats de l'étude confirment les conclusions de Monteroi (1992) et de Fall (2004), selon lesquelles la construction d'un barrage anti-sel modifie la composition chimique des eaux. Selon Fall (2004) et Fermont (2002), la mise en place du barrage a provoqué une baisse considérable de la nappe phréatique et entraîné la chute du potentiel hydrogène $(\mathrm{PH})$ du marigot. D'ailleurs, Keita (2004) montre qu'en 1988, la salinité était de 39g/l avant la construction du barrage et de 9,3g/l après son installation à Affiniam. La présente étude a permis de montrer que le barrage contribue à la baisse de la salinité des eaux aussi bien en amont qu'en aval pendant la saison des pluies jusqu'en début de saison sèche. Cependant, à la fin de la saison sèche, la salinité augmente de manière drastique dans la partie aval pour atteindre des valeurs jamais égalées dans le marigot de Bignona avant la construction du barrage. D'ailleurs, ces fluctuations expliqueraient le pic de $120 \mathrm{~g} / \mathrm{l}$ noté en aval du barrage en fin de saison sèche. Ce pic pourrait aussi s'expliquer par la présence de dépressions inondées longtemps soumises à l'évaporation sans contact avec les eaux de la marée haute. Au plan de l'acidité des eaux de surface, l'étude ne permet pas d'expliquer les raisons d'un $\mathrm{pH}$ en moyenne quasi-neutre dans la zone. Un suivi séquentiel de ce paramètre pour l'eau et le sol, au moins deux ans, pourraient permettre de mieux expliquer cette tendance évolutive du $\mathrm{pH}$. En tout état de cause, selon IBGE (2005) un $\mathrm{pH}$ qui varie entre 6,5 et 8,5 dans un étang est favorable à la vie aquatique. Cependant, il reste d'autres paramètres à évaluer dans ce milieu notamment la température, le taux d'oxygène et la conductivité électrique des eaux avant de pouvoir se prononcer sur les possibilités de développement de l'aquaculture en amont du barrage. Le cas échéant, de telles conditions favorables pourraient constituer le levier de 
mesures adaptatives pour les populations affectées par la perte des valeurs écologiques et socioéconomique de la mangrove de leur terroir. Quels que soient les études et les résultats qui seront obtenus, l'état sénégalais gagnerait à mieux gérer le barrage d'Affiniam pour amenuiser les dégâts constatés sur le terrain. Aujourd'hui, le barrage fait partie intégrante de l'écosystème mangrove, la recherche de solutions s'impose pour minimiser les inconvénients de l'ouvrage. N'est-il pas temps de former les populations à la gestion du barrage, de les responsabiliser et de les suivre jusqu'à la maîtrise des opérations techniques d'ouverture et de fermeture des vannes? A partir de ce moment-là, dans le domaine de la riziculture, pourrait être mise à profit par les autochtones expérimentés.

Effets sur la composition floristique, densité et structure de la végétation

Au plan de la diversité, six (6) espèces de palétuviers sont présentes comme c'est le cas au Delta du Saloum selon divers auteurs (Ndour, 2005, Ndour et al., 2013). Cependant, seules Avicennia germinans et Rhizophora mangle sont rencontrées en amont du barrage. Par ailleurs, il ressort que la mangrove du marigot de Bignona est pauvre en espèces par rapport à la mangrove de l'Afrique de l'Est qui compte dix espèces au Kenya (Geoffrey, 2009). Concernant la flore herbacée, les espèces compagnes rencontrées sont Sesuvium portulacastrum et Philoxerus vermicularis. Entre autres espèces herbacées, il y'a Nymphoïdes indica, Nymphaea lotus, Fuirena ciliaris et Imperata cylindrica dont la présence en amont du barrage est favorisée par le stockage des eaux douces. L'analyse de la composition floristique montre ainsi la coexistence entre les palétuviers et certaines espèces d'eau douce dans la partie amont. Les résultats de l'étude permettent de conclure que la modification du régime hydrologique du marigot de Bignona a induit un changement des conditions environnementales qui compromet le processus de la régénération des palétuviers et favorise le développement d'autres espèces d'eau douce en amont du barrage. D'ailleurs, l'étude a aussi montré que la densité de la végétation est actuellement plus importante en aval (3869 individus/ha) qu'en amont (245 individus/ha). Par ailleurs, la régénération naturelle de la mangrove est seulement notée en aval du barrage où les populations participent à la restauration de l'écosystème en plantant des palétuviers. Au plan de la physionomie de la végétation, la structure des palétuviers est discontinue dans la zone. En amont, l'un des faits marquants de cette déstructuration reste l'absence de la régénération naturelle et la présence de rares individus de $R$. mangle et Avicennia germinans. Cette nouvelle structure de la mangrove témoigne d'une régression plus intense des palétuviers dans la partie amont.

\section{Conclusion}

La présente étude a permis de comprendre la dynamique de la mangrove autour du barrage d'Affiniam. La construction du barrage était, à priori, une solution pour protéger les terres contre la salinisation et la satisfaction des besoins alimentaires en riz des populations. Cependant, la mangrove a perdu des superficies importantes de palétuviers après sa construction et la riziculture n'est toujours pas développée autour du barrage. En effet, la superficie de la mangrove était de 4203,99 ha en 1986 et de 1867,68 ha en 2010. Ces statistiques indiquent un recul important de la superficie de mangrove. Au plan de la dynamique de la mangrove, la régénération naturelle est seulement notée en aval avec une densité de 2154 individus/ha. Le stockage des eaux douces en amont a conduit au développement d'espèces herbacées d'eau douce. Cette nouvelle dynamique témoigne de la mise en place progressive d'un nouveau type d'écosystème dans le milieu. Au plan physico-chimique, la salinité et le $\mathrm{pH}$ des eaux du marigot sont plus élevés en aval qu'en amont.

La situation actuelle, marquée par la diminution de la superficie de la mangrove et des terres rizicoles, la baisse des ressources halieutiques dans le marigot, a entraîné le 
développement d'activités telles que l'exploitation forestière et le maraîchage qui autrefois étaient moins pratiquées. Ces activités sont devenues essentielles, permettant aux populations riveraines d'améliorer leurs revenus. Il serait donc important de mieux gérer le barrage dans un but de régulation les plans d'eau entre les parties amont et aval pour la sauvegarde de la mangrove. Il serait aussi nécessaire de développer des stratégies permettant de restaurer la mangrove tout au long du marigot pour le retour de ses fonctions écologique et socioéconomique dans la zone. A cet égard, une étude du sol et de ses eaux interstitielles pourrait permettre d'évaluer les possibilités de plantation d'espèces halotolérantes en amont du barrage. Il serait également important de réévaluer les potentialités rizicoles autour du barrage puisque le barrage n'a pas permis jusqu'à présent le développement de la riziculture. Les réponses à ces questions constitueraient des informations complémentaires aux résultats de la présente étude. Elles pourraient aussi contribuer à une meilleure adaptation des populations aux changements environnementaux observés autour du barrage.

\section{CONFLITS D'INTERETS}

Les auteurs déclarent que le présent article ne fait l'objet d'aucun conflit d'intérêt.

\section{CONTRIBUTIONS DES AUTEURS}

MT est l'investigatrice principale. Elle a dirigé les travaux d'inventaire de la mangrove et assuré le traitement des données. $\mathrm{NN}$, BS ont été les initiateurs du projet. Ils ont orienté et supervisé les travaux. MD a contribué activement à la correction de l'article. AA a contribué à la rédaction de la méthodologie de recherche, particulièrement dans les méthodes de traitement et d'analyse des images satellites.

\section{REMERCIEMENTS}

Les auteurs remercient l'OCEANIUM pour son appui technique et son soutien moral dans la réalisation du mémoire d'où est extrait le présent article. Ils remercient l'ensemble des géographes qui ont contribué à la réussite de ce travail.

\section{REFERENCES}

Albergel J, Brunet D, Dubée G, JP Montoroi, Zante P. 1991. Gestion d'un barrage antisel en Basse Casamance (Sénégal). In Utilisation Rationnelle de l'Eau des Petits Bassins Versant en Zone Aride, Kergreis A, Claude J (éds). Ed. AUPELFUREF : Paris, France ; 275-285.

ANSD. 2013. Agence Nationale de la Statistique et de la Démographie Situation économique et sociale de la région de Ziguinchor. Rapport régional. Service Régional de la Statistique et de la Démographie de Ziguinchor, Avril 2013, $126 \mathrm{p}$.

Assani AA, Petit F. 2004. Impact of hydroelectric power release on the morphology and sedimentology of the bed of the WArche River (Belgium). Earth Surface Processes and Landforms, 29: 133-143.

AUOF. 2000. Préparation du contrat de la commune de Bignona-rapport final CABEX-SARL, 4 Août 2000. 122p.

Barry B, Boivin P, Brunet D, Montoroi JP, Mougenot B, Touma J, Zante P. 1988. Evolution des stratégies d'aménagements hydro-agricoles des sols salés en basse Casamance. In GAYE CB (éd), Deuxième journée de l'eau au Sénégal, recueil des communications, Dakar, Sénégal, université Cheikh Anta Diop/DEH, p. 104-117.

Bassène Y. 2010. Plan d'aménagement et de développement pour un terroir traversé par un conflit armé : communauté rurale de Nyassia, mémoire de Master, Université Cheikh Anta Diop de Dakar UCAD, Dakar, 69p.

Bérhaut J. 1967. La Flore du Sénégal. Claire Afrique ; 485p. 
Brandt SA. 2000a. Classification of geomorphological effects downstream of dams. Catena, 40: 375-401.

Brandt SA. 2000b. Prediction of downstream geomorphological changes after dam construction : a stream power approach. Water Resources Developpement, 16(3): 343-367.

CSE. 2010. Centre de Suivi Ecologique. Evaluation des conditions et tendance des Ecosystèmes Forestiers et de leurs Services au Sénégal, Rapport final PASEF_CSE Octobre 2010, 244p.

Dasylva M. 2013. Agriculture urbaine souspluie et sécurité alimentaire dans la commune de Ziguinchor, mémoire de master en AGDEFA, UFR-ST, Université Assane Seck de Ziguinchor, Ziguinchor, $52 \mathrm{p}$.

Daube DD, Hanrahan DR, Geist, Parseley MJ. 2003. Impacts of the columbia Rivers hydroelectric system on main-stem habitats of fall Chinook salmon. North American Journal of Fisheries Management, 23(3): 641-659.

Dieye E H B, Diaw A T, Sané T, Ndour N. 2013. Dynamique de la mangrove de l'estuaire du Saloum (Sénégal) entre 1972 et 2010, Cybergeo : European Journal of Geography [En ligne], Environnement, Nature, Paysage, document 629, mis en ligne le 09 janvier 2013, consulté le 25 mars $2016 . \quad$ URL : http://cybergeo.revues.org/25671, DOI.

Dieye B, Sané T, Ndour N, SY O. 2015. Dynamique de la mangrove et impacts dans le Département d'Oussouye (Basse Casamance) entre 1972 et 2014, Université Assane SECK de Ziguinchor. Rapport de projet, 41p.

Diouf. 2013. Ouvrages hydrauliques et modèle de gestion de l'eau dans le bassin du fleuve Casamance (Sénégal). Thèse de doctorat en Géographie humaine, économique et régionale, Paris 10, 309p.

Fall M. 2004. Les Impacts Socio-économiques et Environnementaux du barrage Anti-Sel D’Affiniam, Mémoire de fin d'étude du second cycle, Département d'Aménagement Du Territoire, Environnement et Gestion Urbaine (ATEGU). Ecole Nationale d'Economie Appliquée (ENEA), Dakar, Sénégal, 57p.

Fermont AM. 2001. La riziculture et la dégradation des sols dans la vallée de Bignona, au Sénégal Rapport du projet WARDA/FDPDE, 85p.

Geoffrey MR. 2009. Les activités humaines, la menace principale pour la riche forêt de mangrove du Delta du fleuve Tana au Kenya. Revue Nature \& Faune, 1(24): 73-77.

IBGE. 2005. Institut Bruxellois pour la Gestion de l'Environnement, Qualité physico-chimique des eaux de surface; cadre général, données de l'IGBE : l'eau à Bruxelles, 16p.

Keita B. 2004. Rapport d'activités du barrage d'Affiniam, 24p.

Manga BA, Tendeng M. 2010. Etat des lieux de la mangrove et des activités socioéconomiques dans la zone d'influence du barrage d'Affiniam. Mémoire de fin d'études du premier cycle. Université Assane Seck de Ziguinchor, Ziguinchor, 47p. ;

Monteroi JP, Zante P. 1989. La mise en valeur des terres dégradées par la salinisation en basse Casamance (Sénégal). Séminaire «Estudio de las relacionesagua-suelovegetacion y ganado en la zona aridadelnorte de Mexico. Orientado a la utilizacionracional de ebtosrecursos», 2327 octobre 1989, Mexico. 12p.

Montoroi JP. 1992. Les sols et l'agriculture dans le domaine estuarien de BasseCasamance. In Conservation et Utilisation Durable des Ressources Naturelles du Bassin Hydrographique de la Casamance, Grepin G, Pomerleau C, Pirot JY (Eds). AJACZG/ISRNORSTO.VI/UICN, ZiguinchorDakar: 52-59.

Monteroi JP. 1996. Mise en valeur des basfonds en Basse-Casamance (Sénégal); O.R.S.T.R.O.M, Laboratoire des 
formations superficielles, Fonds documentaires, Agriculture et développement $\mathrm{n}^{\circ} 10$ - Juin 1996, 73 pages.

Ndao M. 2012. Dynamiques et gestion environnementales de 1970 à 2010 des zones humides au Sénégal : Etude de l'occupation du sol par télédétection des Niayes avec Djiddah Thiaroye Kao (à Dakar), Mboro (à Thiès et Saint-Louis). Doctorat de l'Université de Toulouse. $370 \mathrm{p}$.

Ndiaye 2003. Le fleuve Sénégal et les barrages de l'OMVS: quels enseignements pour la mise en ouvre du NEPAD? Revue Electronique en Sciences de l'Environnement, Vertigo 4(3).

Ndiaye A. 2004. Dynamique fluvial et évolution morphosédimentaire de l'estuaire du fleuve Sénégal après la mise en eau du barrage de Diama. Thèse de doctorat de $3^{\text {ème }}$ cycle. Option Environnement Sédimentaires, Université Cheikh Anta Diop de Dakar UCAD, Dakar, 149p.

Ndour N. 2005. Caractérisation et étude de la dynamique des peuplements de mangrove du Delta du Saloum (République du Sénégal). Dakar, Faculté des Sciences et techniques, Thèse de troisième cycle, Université Cheikh Anta Diop de Dakar, Dakar 180 p.

Ndour N, Sambou B, Dieng DS. 2013. Production de litière de la mangrove du
Parc National du Delta du Saloum (Sénégal). Int. J. Biol. Chem. Sci., 7(6) : 1-2.

Niasse M, Afouda A, Amani A. 2004. Réduire la vulnérabilité de l'Afrique de l'Ouest aux impacts du climat sur les ressources en eau, les zones humides et la désertification, Eléments de stratégie régionale de préparation et d'adaptation, UICN-Union mondiale pour la nature, Gland, Suisse et Cambridge, RoyaumeUni. Xviii + 71pp.

Paderca. 2008. Etablissement de la situation de référence du milieu naturel en basse et moyenne Casamance, Rapport final Centre de Suivi Ecologique pour la gestion des ressources naturelles. Juillet 2008. 201 pages.

Rollet AJ. 2007. Etude et Gestion de la Dynamique sédimentaire d'un tronçon fluvial à l'aval d'un barrage : Cas de la basse vallée de l'Ain Thèse de doctorat Université Jean Moulin Lyon 3. 305p.

Schilt CR. 2007. Developping fish passage and protection at hydropower dams. Applied Animal Behaviour Science, 104(3-4): 295-325.

UICN 2009. Union Internationale pour la Conservation de la Nature, Rapport Final les mangroves du Sénégal: Situation actuelle des ressources, leur exploitation et leur conservation, 66p. 